\title{
Beyond Escherichia coli: Synthetic Biology-Focused Platforms and Toolboxes for Engineering other Bacteria
}

\author{
James Ellinger* \\ University of Tokyo, Komaba 3-8-1, Japan
}

Received: June 15, 2016; Accepted: July 12, 2016; Published: July 18, 2016

*Corresponding author: James Ellinger, Assistant Professor, Center for Global Communication Strategies, University of Tokyo, 153-8902, Tokyo Meguro-ku, 3-8-1 Komaba, KIBER, Japan, E-mail: jellinger@aless.c.u-tokyo.ac.jp

\begin{abstract}
Synthetic biology is a field that has rapidly grown in the past decade. Many tools and standardized parts, such as BioBricks ${ }^{\mathrm{TM}}$, are now available that allow scientists to rapidly assemble gene circuits or multi-step pathways. However, one limitation is that the vast majority of parts have been designed and characterized with the goal of using Escherichia coli as the chassis. Yet, other species of bacteria are of interest due to native metabolic properties or tolerance to extreme conditions. Therefore it is necessary to expand the scope of synthetic biology beyond E. coli. This review introduces recently developed platforms and toolboxes for other bacteria, with a slight emphasis towards development of promoter systems.
\end{abstract}

Keywords: Synthetic biology; BioBrick ${ }^{\mathrm{TM}}$; BglBrick; Corynebrick; Methylobrick; Bacillus; Corynebacteria; Ralstonia; Streptococcus, Methylobacterium; Cyanobacteria

\section{Introduction}

The ability to quickly and efficiently build plasmid-based constructs using standardized parts is one of the key tenets of synthetic biology. For example, the BioBrick ${ }^{\mathrm{TM}}$ assembly standard allows researchers to 'snap' together a series of standardized biological parts, such as promoters, Ribosome Binding Sites (RBS), and genes to create complex assemblies (for details see Knight [1] or the introduction of [2]). Furthermore, compositetype parts such as 'Protein Generators' (http://parts.igem.org/ Help:Protein_Generators) can be assembled into a plasmid in succession to create whole pathways. Synthetic biology has been used recently to study bacterial communication [3] and bacterial computing [4]. As well as production of biochemical commodities [5]. The ability to rapidly assemble these circuits is crucial to accelerate synthetic biology research.

However, as is evident from searching the Registry of Standard Biological Parts (http://parts.igem.org), the largest and most accessible online repository of biological parts, the vast majority of bacterial synthetic biology parts were developed for Escherichia coli. Furthermore, a comparison of synthetic biology-related literature pertaining to E. coli, Bacillus subtilis, Corynebacterium glutamicum, Streptomyces, or Cyanobacteria published between 2004 and 2015 shows that publications for $E$. coli eclipse the others by approximately 10-fold (Figure 1).

Although E. coli has proven to be a useful tool for many decades, other bacteria may prove beneficial depending on the desired goal. For example, through native metabolic pathways, Ralstonia eutropha is able to produce polyhydroxyalkanoates [6]. These molecules can be extracted and processed to produce biodegradable plastics. Alternatively, the holy grail of synthetic biology may be to use cyanobacteria such as Synechococcus or Synechocystis to converts $\mathrm{CO}_{2}$ directly to biofuels or biochemical commodities via photosynthesis. For example, Synechococcus elongatus PCC7942 has been used to produce 2,3-butanediol [7].

Recently, a handful of synthetic biology-focused platforms

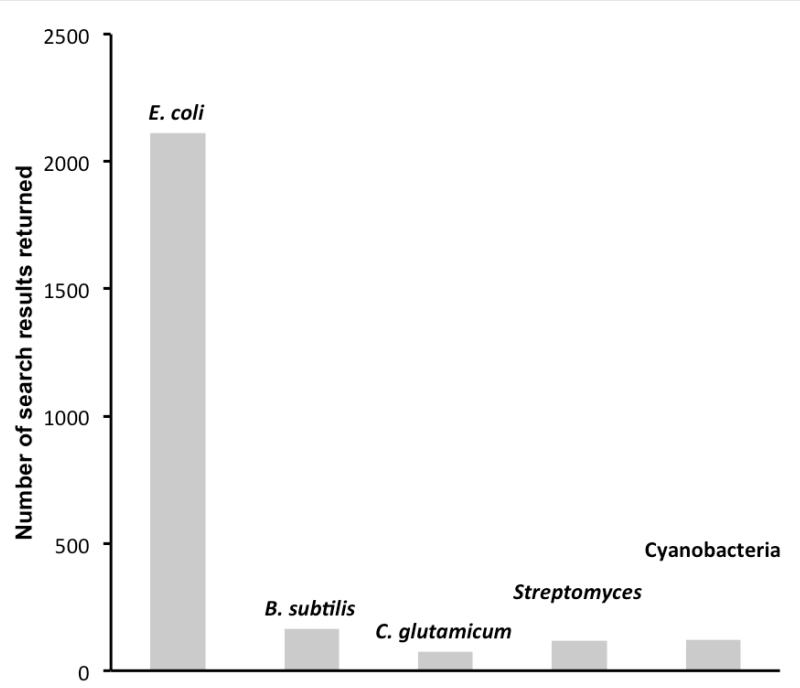

Figure 1: Number of search results returned after using the 'advanced topic search' function from Web of Science (http://www.webofscience. com). The search was conducted between years 2004 and 2015 (roughly corresponding to the modern era of synthetic biology). Specifically, the search was conducted using the following command: TS= ("synthetic biology" AND "yyyy"), TS = topic search; and "yyyy" was a term such as "Escherichia coli". Note that although the abbreviated form is used in the figure, the search term used was the full name, i.e. E. coli was searched as Escherichia coli. Bacterial species and classifications were chosen due to those organisms' status as model organisms, industrial relevance, or pharmaceutical relevance. 
and toolboxes have been developed for various industrially or medically relevant bacteria of various classifications (Table 1).In general, platforms refer to plasmid systems which have been designed to take advantage of easy-to-use assembly standards such as BioBrick ${ }^{\mathrm{TM}}$ or BglBrick, a derivative of the BioBrick ${ }^{\mathrm{TM}}$ system with expanded assembly capability [8]. Furthermore, plasmids also contain an E. coli origin of replication for construction and propagation. Whereas toolboxes focus more on providing characterization or optimization of useful genetic parts such as promoters or RBS, but which do not necessarily follow an assembly standard. This mini-review is presented as a guide to condense the knowledge of these systems into a single resource.

\section{Bacillus subtilis}

Bacillus subtilis, a spore-forming aerobe, is a model organism and was the first Gram-positive to have its full genome sequenced [9]. Furthermore, due to its natural ability to secrete enzymes directly to the extracellular medium, B. subtilis has incredible industrial potential [10]. The Bacillus BioBrick ${ }^{\mathrm{TM}}$ Box developed by Radeck, et al. [11] is a genetic toolbox and platform composed of three integration vectors, a set of six promoters, and a set of five epitope tags that can be used for protein handling or detection. Each integration vector contains a different region of homology as well as a different antibiotic resistance cassette, thereby allowing integration of all three vectors into a single $B$. subtilis strain.

Notably, this work showed that the two inducible promoters, $\mathrm{P}_{\text {lial }}$ and $\mathrm{P}_{x y l A}$, could be induced using inducer concentrations spanning three orders of magnitude with a resulting dynamic response of 300- and 100-fold activity. For applications of synthetic biology where multi-gene pathways are constructed,

Table 1: List of various bacteria, beyond $E$. coli, for which platforms or toolboxes have been developed.

\begin{tabular}{|c|c|c|}
\hline Bacteria & Reference & Brief description \\
\hline Bacillus subtilis & [11] & $\begin{array}{l}\text { BioBrick }{ }^{\mathrm{TM}} \text { compatible plasmids } \\
\text { and toolbox geared toward easy } \\
\text { assembly of genetic constructs }\end{array}$ \\
\hline $\begin{array}{l}\text { Corynebacterium } \\
\text { glutamicum }\end{array}$ & {$[13,14]$} & $\begin{array}{l}\text { BglBrick and BioBrick }{ }^{\mathrm{TM}} \text {-like } \\
\text { systems geared towards easy } \\
\text { assembly of genetic constructs }\end{array}$ \\
\hline Ralstonia eutropha & {$[15,16]$} & $\begin{array}{l}\text { Plasmids and toolboxes(does not } \\
\text { match an assembly standard), } \\
\text { genetic part characterization }\end{array}$ \\
\hline $\begin{array}{l}\text { Streptococcus pneu- } \\
\text { moniae }\end{array}$ & [18] & $\begin{array}{l}\text { BglBrick platform and genetic } \\
\text { part characterization }\end{array}$ \\
\hline $\begin{array}{l}\text { Rhodobacter sphaer- } \\
\text { oides }\end{array}$ & [24] & $\begin{array}{l}\text { BioBrick }^{\mathrm{TM}} \text { compatible platform, } \\
\text { genetic part characterization }\end{array}$ \\
\hline $\begin{array}{l}\text { Methylobacterium } \\
\text { extorquens }\end{array}$ & [17] & $\begin{array}{l}\text { Methylobricks, BioBrick } k^{\mathrm{TM}} \text { - } \\
\text { compatible, toolbox of promoters, } \\
\text { genetic part characterization }\end{array}$ \\
\hline $\begin{array}{l}\text { Synechocystis sp. } \\
\text { strain PCC6803. }\end{array}$ & {$[20-22]$} & $\begin{array}{l}\text { BioBrick }{ }^{\mathrm{TM}} \text { compatible platform } \\
\text { and genetic part characterization }\end{array}$ \\
\hline $\begin{array}{l}\text { Synechococcus sp. } \\
\text { strain PCC } 7002\end{array}$ & [23] & $\begin{array}{l}\text { Toobox of genetic parts with } \\
\text { characterization }\end{array}$ \\
\hline
\end{tabular}

predictable regulation of each gene is absolutely necessary to achieve optimal pathway output. Furthermore, the authors used their system to provide the first detailed analysis of the luciferase reporter system in $B$ subtilis. Plasmid constructs built using this reporter system have been used to study promoters involved in the cell envelope stress response in an engineered strain [12].

\section{Corynebacterium glutamicum}

Corynebacterium glutamicum is an industrially relevant bacterium, which is well known for L-amino acid production. Two platforms have been developed to enable rapid assembly of multi-gene pathways in this bacterium $[13,14]$. In the case of Ravasi, et al. [14]. The plasmid's assembly system is an extension of the BioBrick ${ }^{\mathrm{TM}}$ system, whereas Kang, et al. [13] employ the BglBrick system, which for their specific system was renamed to CoryneBrick.

One highlight of Ravasi, et al. [14] was the use of their platform to investigate combinatorial assemblies of promoters and RBS. Using various pairs of these genetic control elements, they were able to modulate protein expression of the fluorophores eGFP and mCherry [14]. On the other hand, Kang et al. demonstrate the utility of their system by introducing a functional xylose utilization pathway in C. glutamicum through heterologous expression of E. coli xylose isomerase and xylulose kinase [13].

\section{Ralstonia eutropha}

Although fixation of $\mathrm{CO}_{2}$ via photosynthesis is a soughtafter goal in synthetic biology, direct incorporation of $\mathrm{CO}_{2}$ into organic compounds using chemolithoautrophic microbes such as Ralstonia eutropha provide a non-photosynthetic route. Recently developed toolboxes for $R$. eutropha characterized various genetic parts [15,16]. Bi, et al. [15] Developed and characterized three plasmids as part of the R. eutropha platform, although this system was not designed to match an assembly standard.

In particular, transcriptional and translational control elements were characterized. Chemically inducible promoter systems driven by the common inducers xylose, Isopropyl B-D1-Thiogalactopyranoside (IPTG), and anhydrotetracycline were shown to be active, and operate over a range of concentrations. Of note, the promoter $\mathrm{P}_{\text {lacuvs }}$, which is induced by IPTG, was only active when the transformed plasmid expressed galactose permease $\left(l_{a} Y\right)$. Furthermore, of three RBS that were evaluated, the consensus sequence for the E. coli RBS was shown to provide the strongest protein translation capability. Finally, an operon was designed to produce the hydrocarbons pentadecane and heptadecene [15]. By altering the combinations of parts used for constructing the operon, the authors were able to modulate the titer and ratio of hydrocarbons produced.

\section{Methylobacterium extorquens}

Another promising avenue toward renewable production of carbon-based compounds is through the use of methylotrophic bacteria. These bacteria have the unique ability to use reduced carbon substrates such as methanol, which can be derived from natural gas, as the sole source of energy and carbon. Schada von 
Borzyskowski, et al. [17]. Developed a platform and toolbox for synthetic biology applications in the methylotrophic alpha proteobacterium Methylobacterium extorquensAM1 [17]. The platform is BioBrick ${ }^{\mathrm{TM}}$ compatible and was renamed as the Methylobricks system. The Methylobrick platform introduces a set of plasmids that are BioBrick ${ }^{\mathrm{TM}}$ compatible, of which one subset was designed to operate episomally, and the other subset operates by $\mathrm{Tn} 5$-mediated integration into the genome.

As part of their toolbox, the authors evaluated the translational abilities of three constitutive promoters. Interestingly, these promoters are endogenous to M. extorquens AM1. Each promoter conferred a different degree of gene expression ability, thereby allowing researchers to design assemblies with modulated protein output. As a demonstration of the application of their system, the Methylobrick platform and toolbox was used to construct an operon for production of mesaconate using glyoxylate as the feedstock. Additionally, the authors demonstrate that their platform and toolbox is functional in other alpha proteobacteria: Agrobacterium tumefaciens, Caulobacter crescentus, and Paracoccus denitrificans.

\section{Streptococcus pneumoniae}

Although synthetic biology for production of biochemical commodities is one of the most prominent applications, synthetic biology can also be used as a means to support basic research. With a synthetic biology approach, naturally occuring gene circuits can be broken into smaller components or studied under controlled conditions, similar to reverse engineering. The bacterium Streptococcuspneumoniae is a commensal organism that colonizes humans, and while benign under most circumstance, certain environmental cues lead to virulence. Although difficult to study these cues in vitro, a recently developed platform and toolbox using the BglBrick assembly standard provides the option to begin studying $S$. pneumoniae using a synthetic biology approach and thus under controlled conditions [18]. This platform contains components necessary for either chromosomal integration or episomal study in S. pneumoniae.

Within the toolbox, the authors characterized several promoters. A set of synthetic constitutive promoters was developed and shown to modulate expression levels of the luciferase reporter gene over three orders of magnitude. Additionally, a $\mathrm{Zn}^{2+}$-inducible promoter system was developed and shown to modulate expression levels of reporter over three orders of magnitude in a $\mathrm{Zn}^{2+}$ concentration dependent manner. Further development is needed to expand the functionality of this platform and toolbox; however components have been used to study the dynamics of antibiotic resistance in $S$. pneumoniae [19].

\section{Photosynthetic bacteria}

The use of photosynthetic bacteria in synthetic biology might be considered as the holy grail due to their ability to use solar energy for carbon fixation. Synechocystis sp. PCC6803 (hereafter PCC6803) is one of the more highly studied cyanobacteria. The platform developed by Huang, et al. [20] is BioBrick ${ }^{\mathrm{TM}}$ compatible and therefore provides a means to rapidly assemble plasmids for synthetic biology applications [20]. The suite of genetic parts available and tested for PCC6803 includes various promoters, reporter proteins, and protein degradation tags. Evaluation of fluorescent reporter proteins was particularly of interest to ensure that natural pigments did not interfere with reporter output; no effect was observed.

However, the major difficulty regarding genetic manipulation of PCC6803 has been the development of reliable and controllable promoter systems. Extending the work mentioned above, the Anderson collection (http://parts.igem.org/Promoters/Catalog/ Anderson), a library of synthetic constitutive promoters was investigated for PCC6803 [21]. This widely used promoter collection was shown to operate over a wide range in PCC6803. Furthermore, a separate library of synthetic promoters, inducible by anhydrotetracycline, has also shown the ability to regulate translation over a broad range [22]. Taken together, the platform and toolbox of genetic parts is a promising starting point for advancing PCC6803 synthetic biology applications.

A toolbox has also been developed for another model cyanobacterium, Synechococcus sp. PCC 7002 [23]. The goal of this toolbox was to provide tools that can reliably control gene expression. In particular, through a combination of truncation and random mutagenesis, the authors built two sets of orthogonal constitutive promoter libraries that could regulate translation over 3 orders of magnitude. One set was based on the promoter $\mathrm{P}_{\mathrm{cpcB}}$, which originated from PCC6803, the other set was derived from the E. coli $\sigma^{70}$ consensus sequence. Of note is that while the original $\mathrm{P}_{\text {срсв }}$ promoter was sensitive to light, the resulting derivatives were not.

A set of promoters that could be induced by IPTG was also developed [23]. By introducing two lac operators into the $\mathrm{P}_{\text {срсв }}$ derivatives and further optimizing the promoter sequence, the authors were able to construct a system that could regulate expression over a range of 2 orders of magnitude when induced using different concentrations of IPTG. Use of this system required introducing and optimizing expression of the lacI repressor. Finally, the authors demonstrate further ability to modulate protein expression through the design of a set of optimized RBS sequences.

Another well-studied photosynthetic bacterium is the purple, non-sulfur photoheterotrophic Rhodobacter sphaeroides. The first attempt to develop a standardized system was produced by Tikh, et al. [24] through the introduction of a BioBrick ${ }^{\mathrm{TM}}$ compatible expression system [24]. Through this work, a truncated version of the native promoter, $\mathrm{P}_{p u \rho}$ was developed and shown to have similar activity to the full-length version. To demonstrate the effectiveness of the system, the membrane protein proteorhodopsin was expressed at levels comparable to those previously reported for highly optimized strains of $E$. coli. Although this platform is still in its infancy, it presents a starting point for further development of synthetic biology tools for $R$. sphaeroides.

\section{Conclusion}

To date, most bacterial synthetic biology has focused on 
developing tools for E. coli. In this review, synthetic biologyfocused platforms and toolboxes for bacteria from a range of classifications were presented. Notably, there has been an emphasis on promoter characterization and development. This is a natural starting point because regulation of transcription is the first step in protein expression. It is the author's hope that this review inspires and emboldens others to pursue synthetic biology using bacteria beyond $E$. coli.

\section{Acknowledgments}

The author would like to thank Dr. Taichi Takasuka for reading and editing this manuscript.

\section{References}

1. Knight T. Idempotent Vector Design for Standard Assembly of Biobricks 2003.

2. Vick JE, Johnson ET, Choudhary S, Bloch SE, Lopez-Gallego F, Srivastava $\mathrm{P}$, et al. Optimized compatible set of BioBrick ${ }^{\mathrm{TM}}$ vectors for metabolic pathway engineering. Appl Microbiol Biotechnol. 2011;92(6):127586. Doi: $10.1007 /$ s00253-011-3633-4.

3. Scott SR, Hasty J. Quorum Sensing Communication Modules for Microbial Consortia. ACS Synth Biol. 2016.

4. Rubens JR, Selvaggio G, Lu TK. Synthetic mixed-signal computation in living cells. Nat Commun. 2016;7:11658. Doi: 10.1038/ncomms11658.

5. Lian J, Zhao H. Reversal of the $\beta$-Oxidation Cycle in Saccharomyces cerevisiae for Production of Fuels and Chemicals. ACS Synth Biol. 2015;4(3):332-41. Doi: 10.1021/sb500243c

6. Peoples OP, Sinskey AJ. Poly-beta-hydroxybutyrate (PHB) biosynthesis in Alcaligenes eutrophus H16. Identification and characterization of the PHB polymerase gene (phbC). J Biol Chem. 1989;264(26):15298303.

7. Oliver JW, Machado IM, Yoneda H, Atsumi S. Cyanobacterial conversion of carbon dioxide to 2,3-butanediol. Proc Natl Acad Sci U S A. 2013 ;110(4):1249-54. Doi: 10.1073/pnas.1213024110.

8. Anderson JC, Dueber JE, Leguia M, Wu GC, Goler JA, Arkin AP, et al. BglBricks: A flexible standard for biological part assembly. J Biol Eng. 2010;4(1):1. Doi: 10.1186/1754-1611-4-1

9. Kunst F, Ogasawara N, Moszer I, Albertini AM, Alloni G, Azevedo V, et al. The complete genome sequence of the Gram-positive bacterium Bacillus subtilis. Nature. 1997;390(6657):249-56.

10. Westers L, Westers H, Quax WJ. Bacillus subtilis as cell factory for pharmaceutical proteins: a biotechnological approach to optimize the host organism. Biochim Biophys Acta. 2004;1694(1-3):299-310.

11. Radeck J, Kraft K, Bartels J, Cikovic T, Dürr F, Emenegger J, et al. The Bacillus BioBrick Box: generation and evaluation of essential genetic building blocks for standardized work with Bacillus subtilis. J Biol Eng 2013;7(1):29. Doi: 10.1186/1754-1611-7-29.
12. Höfler C, Heckmann J, Fritsch A, Popp P, Gebhard S, Fritz G, et al Cannibalism stress response in Bacillus subtilis. Microbiology. 2016;162(1):164-76. Doi: 10.1099/mic.0.000176.

13. Kang MK, Lee J, Um Y, Lee TS, Bott M, Park SJ, et al. Synthetic biology platform of CoryneBrick vectors for gene expression in Corynebacterium glutamicum and its application to xylose utilization. Appl Microbiol Biotechnol. 2014 ;98(13):5991-6002. Doi: 10.1007/ s00253-014-5714-7.

14. Ravasi P, Peiru S, Gramajo H, Menzella HG. Design and testing of a synthetic biology framework for genetic engineering of Corynebacterium glutamicum. Microb Cell Fact. 2012;11:147. Doi: 10.1186/1475-2859-11-147.

15. Bi C, Su P, Müller J, Yeh YC, Chhabra SR, Beller HR, et al. Development of a broad-host synthetic biology toolbox for Ralstonia eutropha and its application to engineering hydrocarbon biofuel production. Microb Cell Fact. 2013;12:107. Doi: 10.1186/1475-2859-12-107.

16. Li H, Liao JC. A Synthetic Anhydrotetracycline-Controllable Gene Expression System in Ralstonia eutropha H16. ACS Synth Biol. 2015;4(2):101-6. Doi: 10.1021/sb4001189.

17. Schada von Borzyskowski L, Remus-Emsermann M, Weishaupt R, Vorholt JA, Erb TJ. A Set of Versatile Brick Vectors and Promoters for the Assembly, Expression, and Integration of Synthetic Operons in Methylobacterium extorquens AM1 and Other Alphaproteobacteria. ACS Synth Biol. 2015;4(4):430-43. Doi: 10.1021/sb500221v.

18. Sorg RA, Kuipers OP, Veening JW. Gene Expression Platform for Synthetic Biology in the Human Pathogen Streptococcus pneumoniae. ACS Synth Biol. 2015;4(3):228-39. Doi: 10.1021/sb500229s.

19. Sorg RA, Veening JW. Microscale insights into pneumococcal antibiotic mutant selection windows. Nat Commun. 2015;6:8773. Doi: 10.1038/ ncomms 9773

20. Huang HH, Camsund D, Lindblad P, Heidorn T. Design and characterization of molecular tools for a Synthetic Biology approach towards developing cyanobacterial biotechnology. Nucleic Acids Res. 2010;38(8):2577-93. Doi: 10.1093/nar/gkq164.

21. Camsund D, Heidorn T, Lindblad P. Design and analysis of LacIrepressed promoters and DNA-looping in a cyanobacterium. J Biol Eng. 2014;8(1):4. Doi: 10.1186/1754-1611-8-4.

22. Huang $\mathrm{HH}$, Lindblad P. Wide-dynamic-range promoters engineered for cyanobacteria. J Biol Eng. 2013;7(1):10. Doi: 10.1186/1754-16117-10.

23. Markley AL, Begemann MB, Clarke RE, Gordon GC, Pfleger BF. A synthetic biology toolbox for controlling gene expression in the cyanobacterium Synechococcus sp. ACS Synth Biol. 2015;4(5):595603. Doi: $10.1021 / \mathrm{sb} 500260 \mathrm{k}$

24. Tikh IB, Held M, Schmidt-Dannert C. BioBrickTM compatible vector system for protein expression in Rhodobacter sphaeroides. Appl Microbiol Biotechnol. 2014;98(7):3111-9. Doi: 10.1007/s00253-0145527-8. 